res que o grupo sem tratamento térmico, às 24 horas. No entanto, não se observou qualquer diferenças $(p=0,098)$ ao fim dos 7 dias de armazenamento. Em relação ao Protemp 4, ao fim de 24 horas, os tratamentos wb1 e hd1 conduziram a um $\Delta E$ estatisticamente $(p<0,05)$ mais elevado que o observado no grupo nt, mas não se observaram diferenças estatisticamente significativas ( $p>0,05)$ entre os grupos mw1 e wb5 e o grupo nt. Aos 7 dias, os tratamentos wb1 e hd1 obtiveram um $\Delta \mathrm{E}$ maior $(\mathrm{p}<0,05)$ que $n t$, e o wb5 apresentou valores de $\Delta E$ menores $(p=0,042)$ que todos os restantes grupos experimentais.

Conclusões: O Protemp 4 apresentou uma maior estabilidade cromática que o Structur 3. Apesar de não se terem verificado diferenças entre os tratamentos para o Structur 3, a imersão em água a $60^{\circ} \mathrm{C}$ durante 5 minutos permitiu obter uma maior estabilidade cromática do Protemp 4. http://doi.org/10.24873/j.rpemd.2018.11.357

\#124 Resistência à flexão do bis-acrílico: efeito do tratamento térmico pós-polimerização CrossMark

Maria Teixeira Santos*, Daniela Lourenço, Sandrine Almeida, Cristina Bettencourt Neves, Bruno Seabra, Jaime Portugal

Faculdade de Medicina Dentária da Universidade de Lisboa

Objetivos: Avaliar a influência do tratamento térmico pós-polimerização na resistência à flexão (RF) de duas resinas bis-acrílicas.

Materiais e métodos: Foram fabricados um total de 100 espécimes em resina bis-acrílica, 50 de Structur 3 (S3) e 50 de Protemp 4 (P4), de forma paralelepipédica com dimensões de $25 \times 2 \times 2 \mathrm{~mm}$, de acordo com as normas ISO 4049:2000. Os espécimes foram aleatoriamente distribuídos por 10 grupos experimentais $(n=10)$ e, imediatamente após o fabrico, sujeitos ao respetivo tratamento térmico: sem tratamento térmico pós-polimerização (nt); sujeitas ao calor produzido por um secador de cabelo convencional com 2200W a uma distância de $20 \mathrm{~cm}$ durante 1 minuto (hd); colocação em micro-ondas a 750W durante 1 minuto (mw); imersão em banho de água a $60{ }^{\circ} \mathrm{C}$ durante 1 minuto (wb1); imersão em banho de água a $60 .^{\circ} \mathrm{C}$ durante 5 minutes (wb5). Após o tratamento, os espécimes foram armazenados a seco à temperatura ambiente $\left(20 \pm 2 .{ }^{\circ} \mathrm{C}\right)$ até serem testados. A resistência à flexão foi determinada realizando testes de flexão de 3 pontos, 30 minutos após o início da manipulação. Adicionalmente, foram fabricados 10 espécimes de cada resina bis-acrílica que não foram submetidos a qualquer tipo de tratamento pós-polimerização e foram testados às 24 horas (S3-nt-24 e P4-nt-24) para servir de controlo. Os dados foram analisados estatisticamente com testes ANOVA seguidos de testes post-hoc segundo Tukey e Dunnett (alfa=0,05).

Resultados: Comparando os espécimes testados ao fim de 30 minutos, os 4 tratamentos térmicos testados aumentaram significativamente $(\mathrm{p}<0,001)$ a $R F$ das 2 resinas relativamente ao respetivo grupo de espécimes não sujeitos a tratamento. Para o Structur 3, apenas o S3-wb5 permitiu obter valores estatisticamente semelhantes $(p=0,999)$ ao grupo S3-nt-24. Para o Protemp 4 , não se observaram diferenças estatisticamente significativas na comparação entre P4-nt-24 e os grupos experimentais P4-hd1 $(p=0,442), P 4-m w 1(p=0,410)$ ou $P 4-w b 5(p=0,974)$. As restantes situações experimentais conduziram a valores estatisticamente $(p<0,001)$ menores que o respetivo controlo de 24 horas.

Conclusões: Os tratamentos térmicos pós-polimerização aumentam a RF das duas resinas testadas. Com determinados tratamentos, é possível obter valores de RF aos 30 minutos semelhantes aos obtidos às 24 horas.

http://doi.org/10.24873/j.rpemd.2018.11.358

\section{\#125 Valores L*a*b* na escala VITA Classical por dois espectrofotómetros - estudo in vitro}

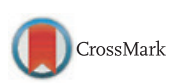

Susana Dias*, Duarte Marques, Ruben Miguel Nunes Pereira, Beatriz Ferreira Fernandes, João Silveira, António Mata

GIBBO-LIBPhys FCT UID/FIS/04559/2013,

Faculdade de Medicina Dentária da Universidade de Lisboa

Objetivos: Determinar e comparar os valores $\mathrm{L}^{*} \mathrm{a}^{*} \mathrm{~b}^{*}$ de dois instrumentos de determinação de cor dentária por espectrofotometria, na medição da escala VITA Classical (VC).

Materiais e métodos: Foram testados dois espectrofotómetros, Spectroshade Micro (SS) eVITA Easyshade (ES), em ambiente controlado utilizando uma câmara escura. Foram realizadas 30 medições a cada guia de cor de três escalas VC de lotes diferentes. Para cada medição foi registado um valor L*a*b*, sendo no ES realizada uma conversão de valores LCh para $\mathrm{L}^{*} \mathrm{a}^{*} \mathrm{~b}^{*}$, por fórmulas previamente definidas. Os resultados foram indicados sob a forma de média e desvio padrão para L*a*b*, de cada uma das guias nos dois espetrofotómetros, e delta $E(\Delta E)$ entre os mesmos. Os resultados foram analisados com recurso ao teste t-Student independente, com nível de significância de $\alpha=0,05$.

Resultados: Foram realizadas 30 medições para cada uma das 16 guias de 3 escalas de lotes diferentes, correspondendo a 1440 medições por espectrofotómetro. De todas as guias de cor avaliadas, apenas não existiram diferenças estatisticamente significativas entre os dois dispositivos para a guia D3 na componente $b^{*}$. Embora medindo a mesma guia, foram detetadas diferenças de $\Delta \mathrm{E}$ entre aparelhos acima do limite considerado como percetível ao olho humano $(\Delta \mathrm{E}>=3,7)$, para as guias B1 $(\Delta \mathrm{E}=3,7 \pm 0,9), \mathrm{B} 2(\Delta \mathrm{E}=6,0 \pm 2,0), \mathrm{A} 2(\Delta \mathrm{E}=3,8 \pm 1,0)$ e $\mathrm{D} 4$ $(\Delta E=3,8 \pm 0,5)$, com uma média global de $\Delta E=3,1 \pm 1,5$.

Conclusões: Embora estes aparelhos apresentem níveis elevados de sensibilidade e especificidade, quando avaliados para a componente $\mathrm{L}^{*} \mathrm{a}^{*} \mathrm{~b}^{*}$ e de acordo com os resultados obtidos, existe uma elevada variabilidade inter-aparelho cujo o impacto clínico deverá ser aferido em estudos in vivo.

http://doi.org/10.24873/j.rpemd.2018.11.359

\#126 Translucidez de diferentes espessuras de zircónia - estudo preliminar

Ana Filipa Pereira*, Luís Proença, Alexandra Pinto, Inês Carpinteiro, Ana Cristina Azul, Inês Caldeira Fernandes

Centro de Investigação Interdisciplinar Egas Moniz; Instituto Universitário Egas Moniz,

Objetivos: A aplicação crescente da zircónia como material estético tem conduzido a uma evolução com melhoria das 